\title{
Experimental determination of viscoelastic properties of a highly compressible porous materials imbibed with water
}

\author{
Minaela Radu ${ }^{1, a}$, Benyebka Bou-Said ${ }^{2}$ and Traian Cicone ${ }^{1}$ \\ 1 Department of Machine Elements and Tribology, University Politehnica of Bucharest, Bucharest, Romania \\ 2 CNRS INSA-Lyon, LaMCoS, Université de Lyon, Villeurbanne, France
}

Received 4 July 2014, Accepted 17 June 2015

\begin{abstract}
The experimental study of a highly compressible porous material, made of cellulose fibers, and imbibed with water, during stress relaxation tests on a rheometer, shows that the material relaxes when kept at constant strain as long as there are open pores. Using the relaxation curves, the behaviour under compression can be divided into two distinct zones, a poro-elastic one and a purely-elastic one, bounded by the zero-porosity limit. In the poro-elastic zone, the "non-hookean" stress-strain curve is fitted using Spriggs' expression of the elastic modulus dependence with porosity. Therefore, the elastic modulus of the porous material is expressed as an exponential function with two parameters: the elastic modulus of the fibers and the initial porosity of the material. For the purely - elastic zone the variation of stress is expressed as a sum of the residual stress obtained from the continuity equation and a Hookean stressstrain variation of a thinner material with the elastic modulus of the fibers - determined previously. The relaxation curves are fitted with a relationship between the decay stress and time, approximated with three terms of Prony series expansion of the generalized Maxwell model.
\end{abstract}

Key words: viscoelasticity / porosity / Spriggs' formula / Young modulus

\section{Introduction}

A new lubrication mechanism, named ex-porohydrodynamic (XPHD) lubrication [1], explains the lifting effect produced by the flow of a fluid through the pores of an extremely compressible porous material subjected to compression. Studies performed recently on the behaviour of such materials under impact, for various contact configurations, reveal good potential in shock absorption. The same observations were established by Weinbaum and coworkers, for porous materials with extremely high porosities imbibed with gases [2,3]. Recently, the research group led by Bou-Saïd has published a series of papers on similar studies related to complex rheological fluid properties and fluid inertia for planar configuration (disk-on-plane) $[4,5]$.

Demo-experiments were done in the laboratory of University Politehnica of Bucharest, on various porous materials imbibed with fluids (water, glycerine, paste, gel) impacted with velocities of $1-2 \mathrm{~m} . \mathrm{s}^{-1}$. The goal of our work is to model the squeeze process and establish the damping capacity of such materials; but first, one should characterize the behaviour of the imbibed material. The present study presents the viscoelastic properties of such complex

\footnotetext{
a Corresponding author: mihaela.radu@upb.ro
}

materials imbibed with water, using low-speed compression tests. In order to establish the elastic modulus of such porous materials, water was used as a fluid - since its viscosity is small, and it will not influence the parameters of the flow. Since porous materials have a changing crosssectional area when compressed, one has to determine the dependence of porosity on the elastic properties.

\section{Experiments description}

The experimental studies on porous materials imbibed with water presented herein are focused to determine, with typical mathematical expressions, the viscoelastic characteristics under compression at low speed.

\subsection{Materials}

This study analyses three materials that presented good damping characteristics under impact in the demoexperiments: Material A and Material B are cellulose sponges, while Material $\mathrm{C}$ is a superabsorbent woven textile (Fig. 1). Samples of diameter $15 \mathrm{~mm}, 22 \mathrm{~mm}$ and $3 \mathrm{~mm}$ imbibed with water were used in different types of 


\section{Nomenclature}

\begin{tabular}{|ll|}
\hline$B$ & Exponent in Spriggs' model $[-]$ \\
$E$ & Elastic modulus of the porous material $[\mathrm{kPa}]$ \\
$E_{0}$ & Elastic modulus of the fibers [kPa] \\
$h$ & Thickness of the compressed material $[\mathrm{mm}]$ \\
$h_{0}$ & Initial thickness of the uncompressed material $[\mathrm{mm}]$ \\
$h_{\mathrm{min}}$ & Thickness of the material that corresponds to zero-porosity $[\mathrm{mm}]$ \\
$t$ & Time [s] \\
$t_{0}$ & Time when the relaxation period begins $[\mathrm{s}]$ \\
$\delta$ & Strain $[-]$ \\
$\delta_{\mathrm{min}}$ & Strain that corresponds to zero-porosity $[\mathrm{mm}][-]$ \\
$\varepsilon$ & Porosity of the material at a given compression $[-]$ \\
$\varepsilon_{0}$ & Initial porosity (uncompressed material) $[-]$ \\
$\lambda_{1,2,3}$ & Stress relaxation times $[\mathrm{s}]$ \\
$\sigma$ & Stress $[\mathrm{kPa}]$ \\
$\sigma_{0}$ & Peak value of the stress at the beginning of the relaxation period $[\mathrm{kPa}]$ \\
\hline
\end{tabular}

Material A

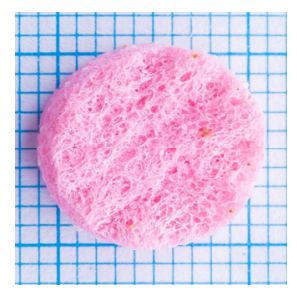

Material B

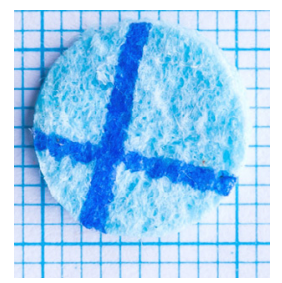

Scale:
Material C

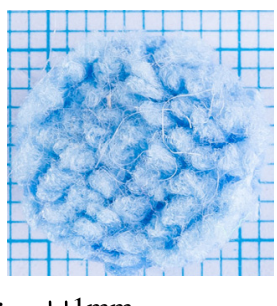

Fig. 1. Macro pictures of the tested materials (in dry condition).

tests. To ensure that the material is well imbibed, it was submersed in water and squeezed in order to remove any air-bubbles inside and then left for $2 \mathrm{~h}$ in water. Different imbibition levels of Material A were tested by weighting the dry sample ( $0 \%$ water) and the fully imbibed in water ( $100 \%$ water), the mass of the water absorbed being calculated as the difference between the two. The material was then squeezed of water and using a digital balance, water was added until it reached the mass of a material with $30 \%$, respectively $60 \%$ of the mass of the maximum quantity of water imbibed calculated previously.

The study focuses mostly on Material A, which was used in all tests presented in Section 3, while Materials B and $\mathrm{C}$ were used only to validate the model presented in Section 3.5.

Material A is a superabsorbent cleaning cloth made of cellulose fibers characterized by open pores. Since the material is brought from a producer of cleaning products, composition or other information are scarce. In Figure 2 is presented a picture of a sample of $12 \mathrm{~mm}$ in diameter in dry and wet condition. The material presents severe swelling after imbibition, especially in thickness, increasing its total volume with $75 \%$.

A SEM (Scanning Electron Microscopy) analysis of the dry material which was previously done [6] shows large and small cavities made of disordered, long fibers of di-
Material A

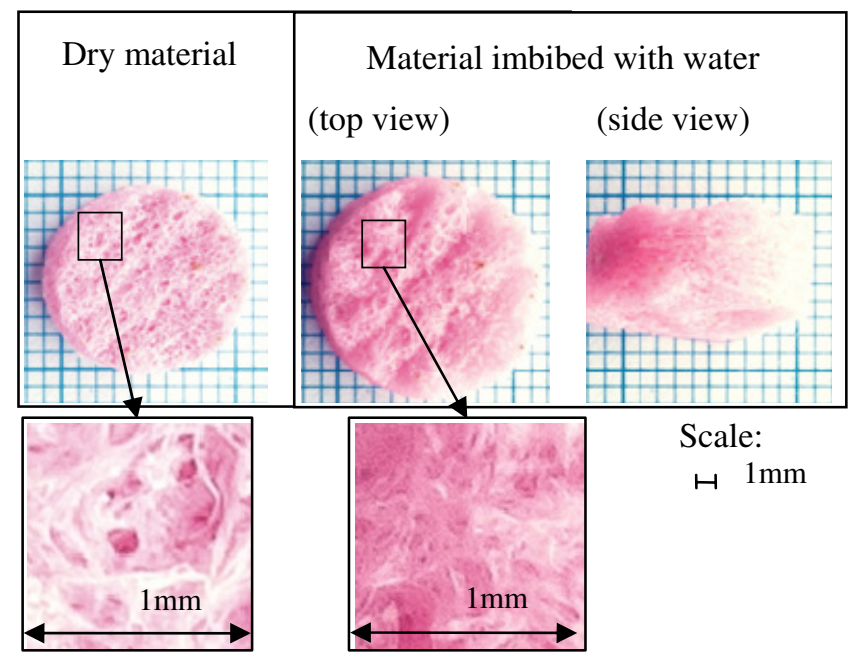

Fig. 2. Macro pictures of Material A in dry and wet condition.

ameters around $10 \mu \mathrm{m}$ to $19 \mu \mathrm{m}$ (Fig. 3). The dimension of the pores varies a lot (from $20 \mu \mathrm{m}$ to $500 \mu \mathrm{m}$ ).

The initial porosity of the materials was measured prior as the ratio of the volume of water absorbed into the material and the total volume of the material, supposing that the water fills every pore of the material. The measurement of the initial porosity $\varepsilon_{0}$ is a very subjective task, therefore one can say that Material A has a porosity between $0.75-0.8$, depending on the level of imbibition of the material.

Under compression, the geometry of the pores changes, squeezing out the water in the material. In this process, we introduce the assumption that the transversal area of the sample does not change and that the fibers of the materials are rigid. Therefore the volume of the solid skeleton is conserved until the porosity becomes zero; resulting in:

$$
h_{0}\left(1-\varepsilon_{0}\right)=h(1-\varepsilon)
$$




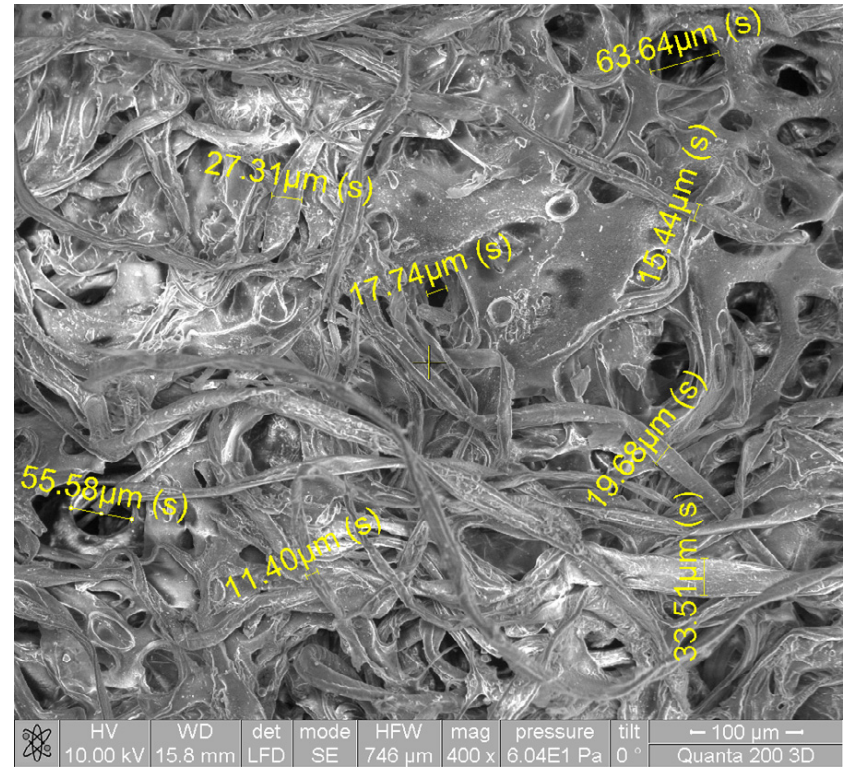

Fig. 3. SEM analysis of dry Material A.

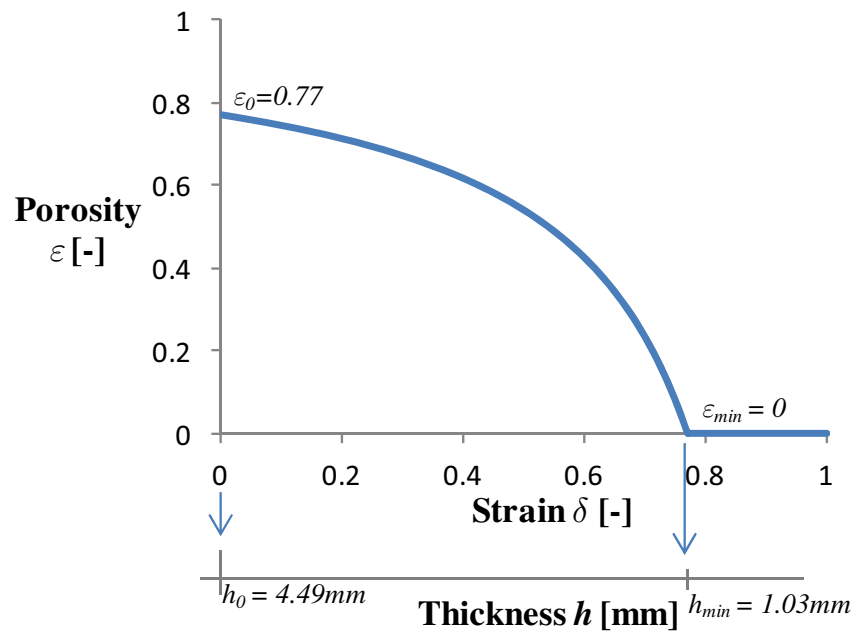

Fig. 4. Porosity variation with respect to strain and thickness for Material A.

where index "0" represents the initial state (uncompressed).

Using the above equation, we are able to extract the expression of the porosity at any state of compression, denoted by $\varepsilon(h)$ :

$$
\varepsilon(h)=1-\frac{h_{0}\left(1-\varepsilon_{0}\right)}{h}
$$

and the value of the thickness of the material at zeroporosity, $h_{\min }$, as:

$$
h_{\min }=h_{0}\left(1-\varepsilon_{0}\right)
$$

The variation of porosity with strain, correspondingly with thickness of the compressed material is presented in Figure 4. When compressing beyond the zero-porosity limit, meaning more than $h_{\text {min }}$, the porosity is zero, so

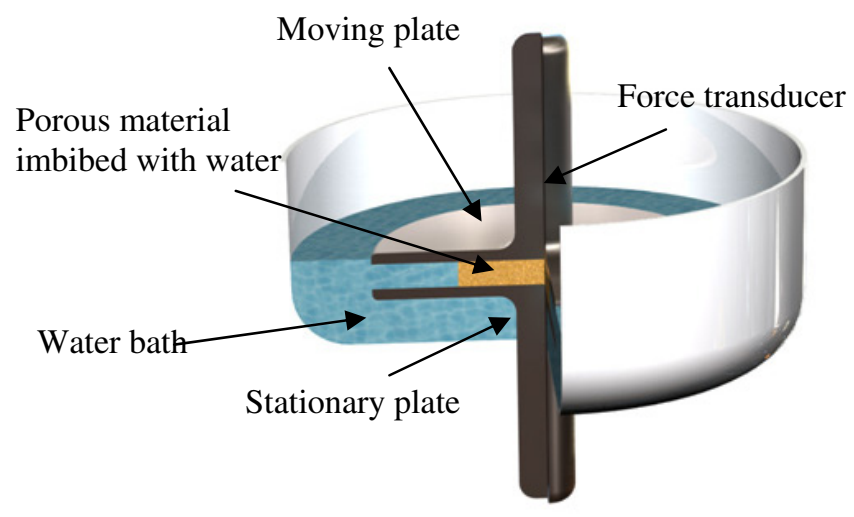

Fig. 5. Experimental set-up of compression tests.

it will be a compact material with no pores. The exact value of initial porosity used in Figure 4 for material A is determined in Section 3.5.

\subsection{Experimental procedure}

The low velocity squeeze tests were done using the rheometer ARES-LS1. The material imbibed with water is positioned between two parallel circular plates (Fig. 5) and compressed with a constant strain rate. The compression was done at room temperature (in a temperature controlled environment $18 \pm 1{ }^{\circ} \mathrm{C}$ ) using different constant velocities; the normal force was recorded, and as a result, the compressive stress was calculated. The force transducer has a normal/axial range of $0.002 \mathrm{~N}-20 \mathrm{~N}$. The probe was fully imbibed with water and immersed in a water bath, except for the case of partially imbibed samples (presented in Sect. 3.4) where no water bath was used.

At the beginning of each experiment, a test of precharge was done to ensure that the material is in contact with the compression plate: when it is in contact with the top surface of the porous material imbibed with water, the force transducer will begin to show a value increase. The thickness of Material A corresponding to a small force of $0.01 \mathrm{~N}$ was found to be $4.49 \mathrm{~mm}$, which was considered the initial thickness of the material $h_{0}$. Water was added at the same level of the material to ensure the full imbibition of the material.

Several types of tests were performed, where the sample was:

- Compressed and immediately uncompressed with a certain velocity.

- Compressed with different velocities and kept over time under a fixed strain.

- Compressed in several steps with pauses of constant strain over time.

The reproducibility of the results was checked by superposition of the stress-strain experimental data for the three samples of different diameters and the results were agreeable (maximum $6 \%$ relative error at any given strain). 


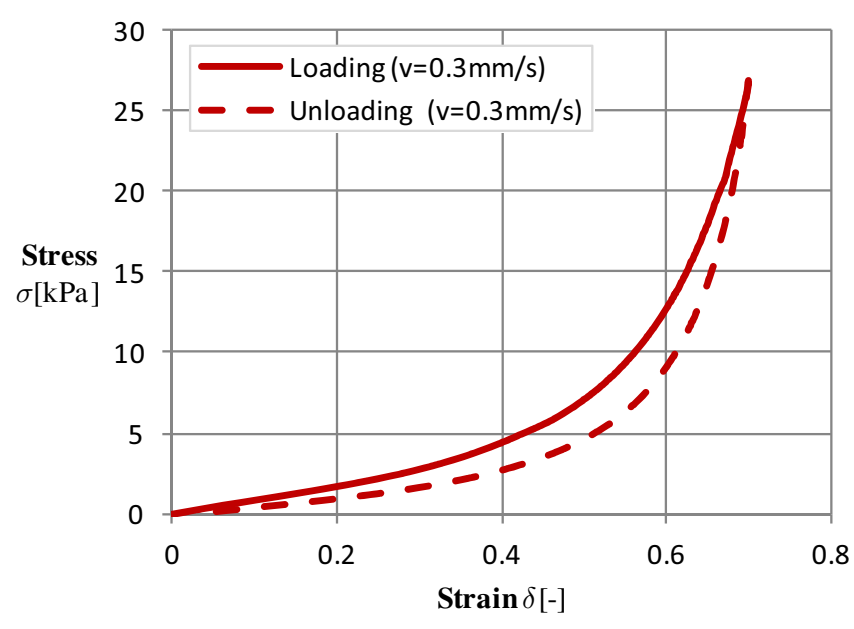

Fig. 6. Stress-strain variation for loading and unloading $M a$ terial A.

\section{Experimental results}

The experiments described in the previous section have led to results that can be analysed independently or in comparison after several criteria. Following are the results that are most important to the authors.

\subsection{Tests regarding the damping capacity}

The damping capability of such a material is demonstrated in the stress versus strain variation which shows that when compressing Material A up to $70 \%$ strain and then immediately uncompressed, a hysteresis curve will appear (Fig. 6). Furthermore, like most of fibrous materials, the selected material shows a nonlinear variation of stress with respect to strain, which means that Hooke law does not apply for this material. That could be because the porosity, and therefore, the geometry of the cross-sectional area, is changing dramatically under compression.

\subsection{Tests regarding the dependence on compression velocity}

Material A was compressed $66 \%$ strain with three different velocities: $0.1 \mathrm{~mm} . \mathrm{s}^{-1}, 0.2 \mathrm{~mm} . \mathrm{s}^{-1}, 0.5 \mathrm{~mm} . \mathrm{s}^{-1}$ and results were very similar (Fig. 7 ). The stress-strain variation does not depend much on speed of compression, but it should be noted that these are low values of compression test speed. Unfortunately the rheometer limitations do not allow faster compression tests. The difference in the values of the stress at the maximum compression is in accordance with the degree of repeatability of the experiments.

The plots in Figure 8 represent the relaxation of the material after the compression tests presented in Figure 7. The normalized stress is defined as the ratio of the stress $\sigma(t)$ to the peak value of stress at the beginning of the

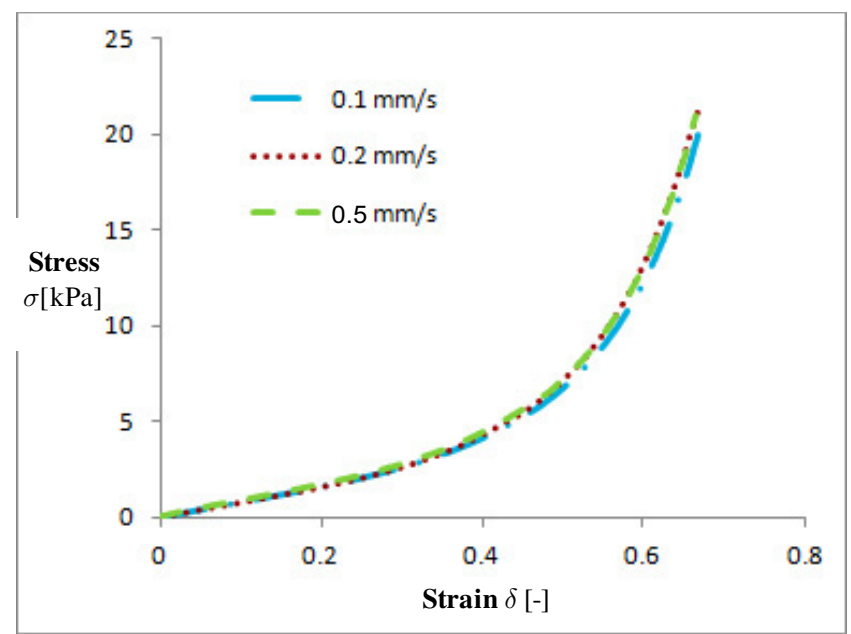

Fig. 7. Stress-strain variation with different speeds of compression of Material A.

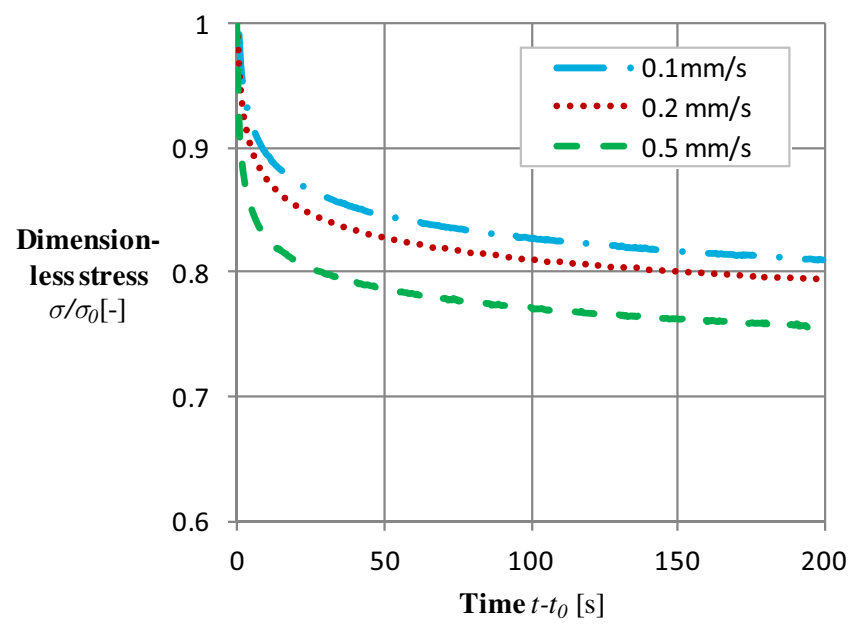

Fig. 8. Dimensionless stress variation in time with different speeds of compression of Material A.

relaxation, $\sigma_{0}$ and it's useful to bring all values of the stress to a common value, 1 . The tests clearly show that the stress relaxes more rapidly after a higher velocity of compression, which can explain the capacity of damping of these materials: the higher is the impact velocity, the faster the material tends to relieve the state of stress.

\subsection{Tests regarding the relaxation stress}

Another type of typical rheological test is to compress the sample in steps, with relaxation pauses after each step. Figure 9 presents a compression test, where the material is compressed in 4 steps for $10 \mathrm{~s}$ with a constant strain rate, each followed by a constant strain pause of $200 \mathrm{~s}$.

The four stress relaxation curves from Figure 9 were normalized by dividing the stress to the peak value at the beginning of the relaxation, $\sigma_{0}$. For comparison purposes, the four curves were translated to the same origin of time 

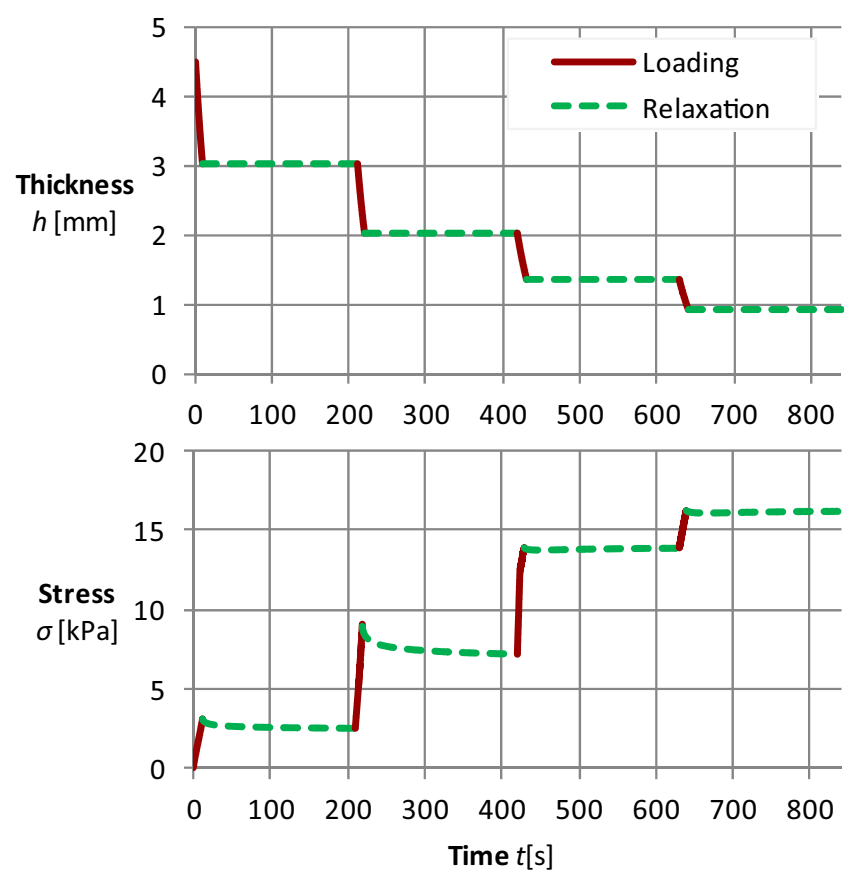

Fig. 9. Four step-compression tests of Material A.

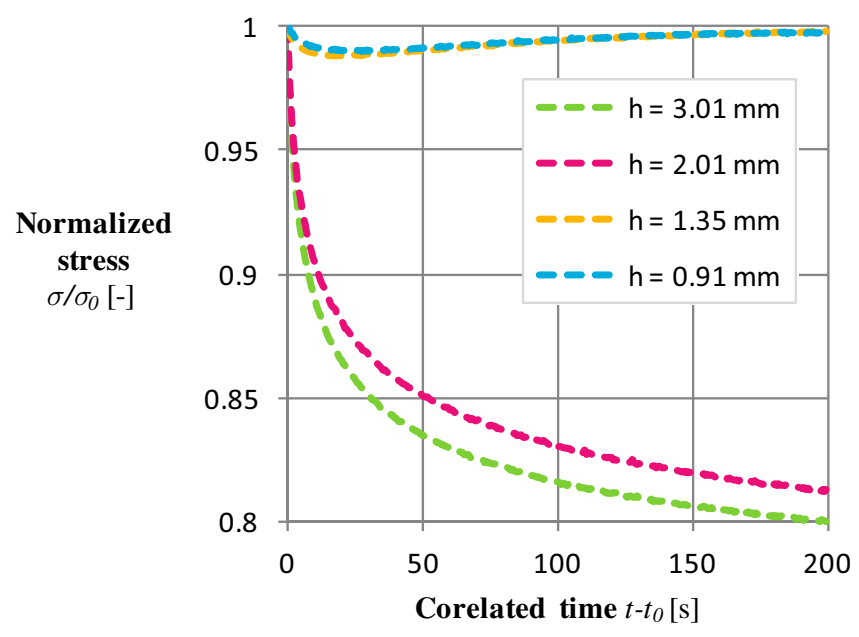

Fig. 10. Normalized relaxation curves of a four stepcompression for Material A.

(Fig. 10). One can observe that the rate of relaxation depends on the gap, due to the porosity and the quantity of water inside. They can be divided into two types of relaxation curves: at low compressions $\left(\delta<\delta_{\min }\right)$ the stress has the tendency to decrease, while at high compressions $\left(\delta>\delta_{\min }\right)$, the stress decreases slightly at the beginning but then it increases and remains constant. The material compressed after $\delta_{\min }$ has an elastic behaviour, which means that it keeps the same level of stress when kept compressed. This limit is associated with the porosity because the material relaxes as long as there are open pores, hence space for the structure of the material to rearrange itself.

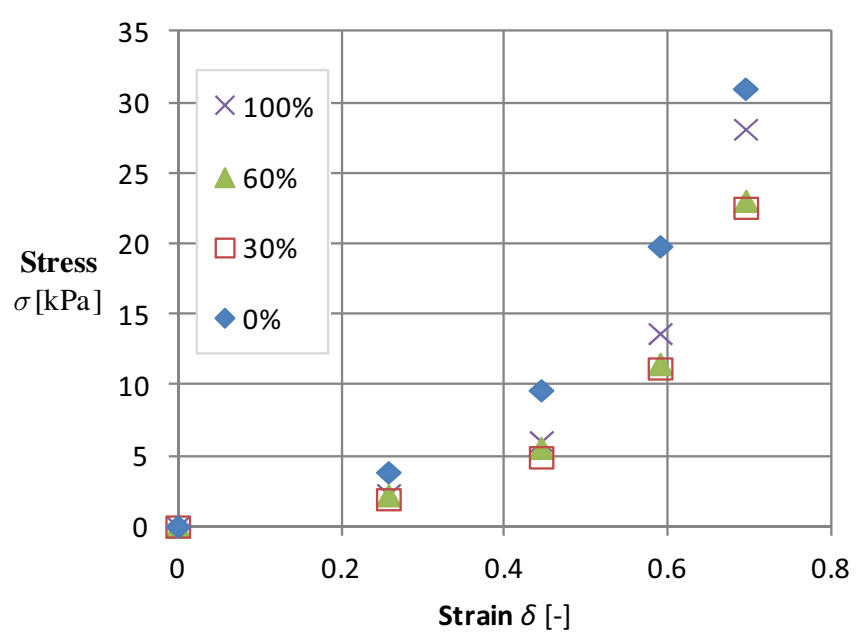

Fig. 11. Stress values obtained from peaks versus strain for Material A.

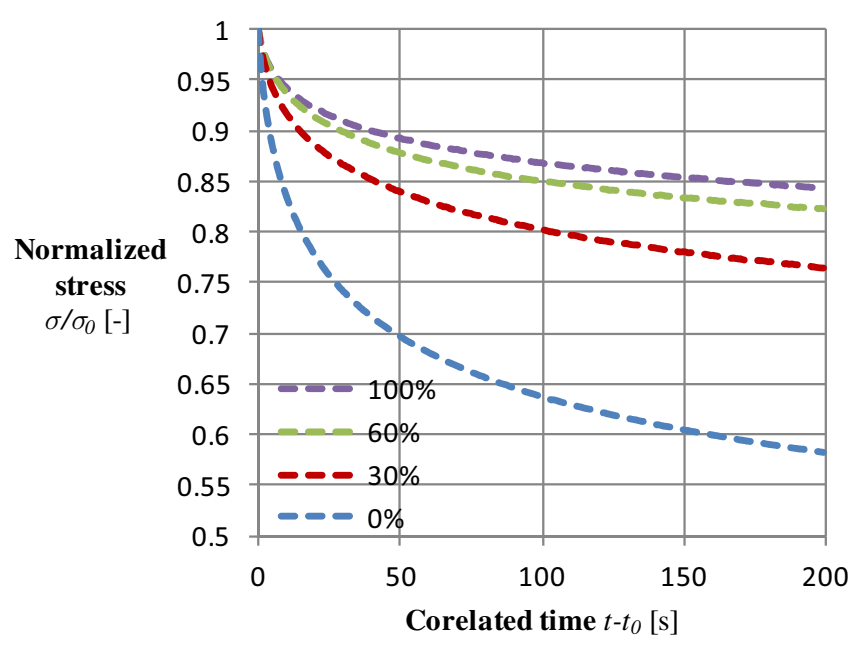

Fig. 12. Relaxation curves for compression until $\delta=0.26$ for different imbibition levels for Material A.

\subsection{Tests regarding the dependence on imbibition level}

The samples of different imbibition levels were compressed in four steps with relaxation pauses of $200 \mathrm{~s}$ between them. In Figure 11 we can observe that the level of imbibition does not influence so much the stress, which is a very useful result in terms of performance - the material does not have to be carefully imbibed. The dry material is more rigid and compact $\left(h_{0}=2 \mathrm{~mm}\right)$ than the imbibed material and has greater values in stress when compressed at the same strains.

The normalized relaxation curves after the first compression $(\delta=0.26$ or $h=3.3 \mathrm{~mm}$ ) from Figure 11 is represented in Figure 12. Since the dry material relaxes, one can say that the relaxation is due to the skeleton of cellulose fibers relaxing in the material and not because of the presence of water. If the material is compressed beyond zero porosity, the response is elastic. The relaxation is actually faster with less water inside the material, in identical compression conditions. 


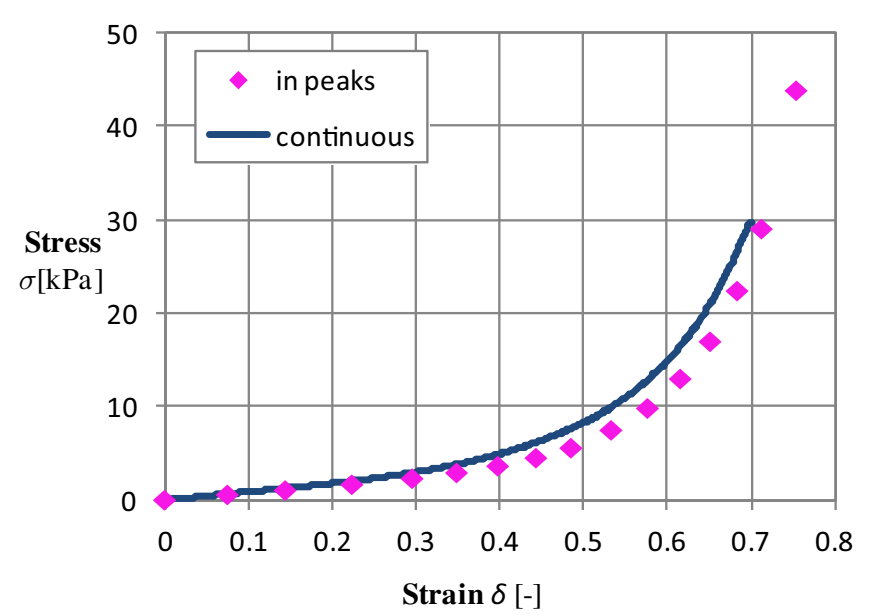

Fig. 13. Stress-strain curve for Material A.

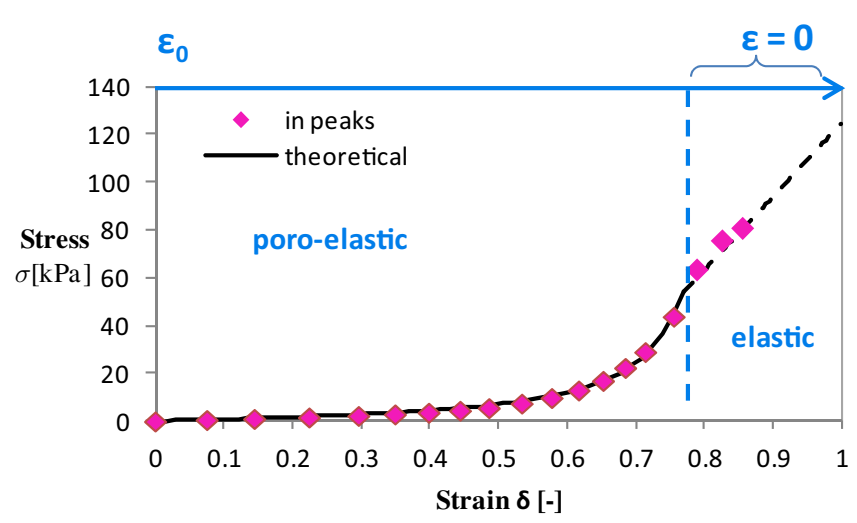

Fig. 14. Experimental curves and theoretical points of stress variation with strain for Material A.

\subsection{Tests regarding the elastic modulus}

A sample of $12 \mathrm{~mm}$ diameter of Material A was compressed in twelve steps with relaxation pauses of $200 \mathrm{~s}$ between steps. The small diameter was chosen in order to compress the material as much as possible in the limit of the force transducer. The values denoted "in peaks" (Fig. 13) are obtained from the highest values of stress $\sigma_{0}$, at the beginning of each of the twelve relaxation curve. Comparing the stresses obtained from a continuous compression of a $35 \mathrm{~mm}$ diameter sample and the ones obtained from this test, one can observe that there is only a slight difference - again considered in the repeatability limit. Therefore, the model of the stress-strain curve can be discussed using the twelve steps experimental data.

The peak values of the twelve steps have an exponential variation with strain until a critic value of the strain - defined by $\delta_{\min }$ (Fig. 14). Following the peak values of stress at high compression strains (after $\delta_{\text {min }}$ ), one can observe a linear variation of the stress-strain curve Therefore, the compression behaviour can be divided into two distinct zones - delimited by the zero-porosity limit $-\delta_{\text {min }}$. This limit can be more precisely determined from the relaxation curves - when the normalized stress is constant, the material was compressed beyond zero-porosity

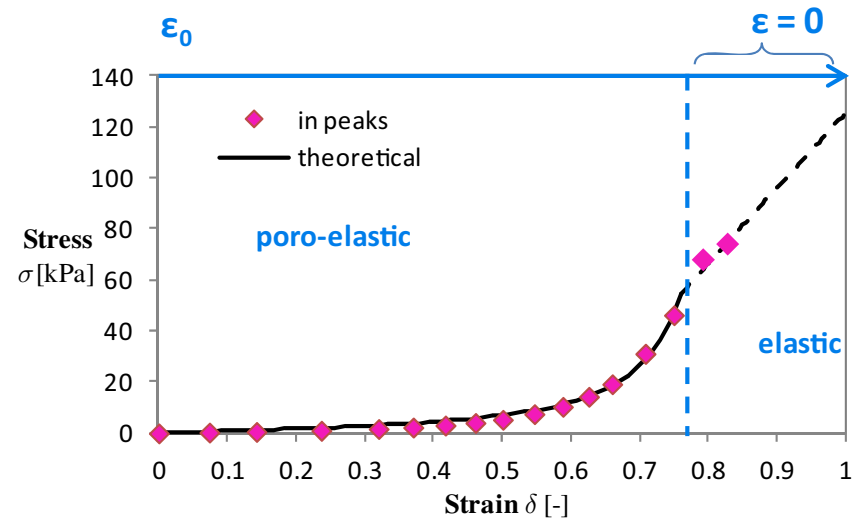

Fig. 15. Experimental curves and theoretical points of stress variation with strain for Material B.

and it behaves as an elastic material. Analytically, it can be divided into 2 curves: an exponential variation and a linear variation.

The exponential variation corresponds to the compression within a positive range of porosity in the interval $\varepsilon \in\left(0, \varepsilon_{0}\right)$ where the geometry of the pores changes with compression. As the initial porosity cannot be estimated very precisely, it was adjusted within the measured range 0.75-0.8, in order to isolate the last three experimental points in the "elastic zone". Therefore, the initial porosity was chosen $\varepsilon_{0}=0.77$.

The law of variation of stress with respect to strain was confirmed as Spriggs' model - an exponential model for porous materials having pores with arbitrary shape [7-9]:

$$
E=E_{0} \mathrm{e}^{-B \varepsilon}
$$

where $\varepsilon$ is calculated according to Equation (2), $E$ is the Young's modulus of porous material, $E_{0}$ is Young's modulus of the fibers. The exponent $B=2.5$ was fitted in order to obtain the best deviation from the experimental points in Figure 14. The effective elastic modulus of the fibers was found as $E_{0}=70.4 \mathrm{kPa}$.

When the material is compressed after the porosity is zero, the material behaves purely elastic. The following equation is built as the sum of a residual stress that comes from the porous material compression (calculated using the continuity equation at $h_{\min }$ ) and a Hookean stress of a "new", thinner sample of thickness $h_{\min }$.

$$
\sigma=E \frac{h_{0}-h_{\min }}{h_{0}}+E_{0} \frac{h_{\min }-h}{h_{\min }}
$$

The two equations describing the variation of stress with strain of extremely compressible porous materials imbibed with water are plotted over the experimental points in Figure 14 - and the fitting is very accurate.

Just for the purpose of validating the model, this model is applied for Materials B and C. Since Material B is very similar with Material A, being also cellulose fibers, the results are very similar and satisfying (Fig. 15).

Material C, is a woven textile and the behaviour in the elastic zone does not fit at all the experimental data (Fig. 16). 
Table 1. The properties of the tested material, as resulted from measurements and fitting of the experimental data.

\begin{tabular}{|c|c|c|c|c|c|c|}
\hline Material & $\begin{array}{l}\text { Increase } \\
\text { in volume }\end{array}$ & $\begin{array}{c}\text { Initial porosity } \\
\qquad \varepsilon_{0}[-] \\
\end{array}$ & $\begin{array}{l}\text { Initial thickness } \\
\qquad h_{0}[\mathrm{~mm}]\end{array}$ & $\begin{array}{l}\text { Minimum thickness } \\
h_{\min }[\mathrm{mm}]\end{array}$ & $\begin{array}{c}\text { Coefficient } \\
\text { in Equation (4) } B[-]\end{array}$ & $\begin{array}{l}\text { Modulus of elasticity } \\
\qquad E_{0}[\mathrm{kPa}]\end{array}$ \\
\hline Material A & $\sim 90 \%$ & 0.77 & 4.5 & 1.03 & 2.5 & 70.4 \\
\hline Material B & $\sim 90 \%$ & 0.76 & 2.8 & 0.67 & 3.2 & 71.2 \\
\hline Material C & $\sim 25 \%$ & 0.65 & 2.1 & 0.73 & 3.6 & 104 \\
\hline
\end{tabular}

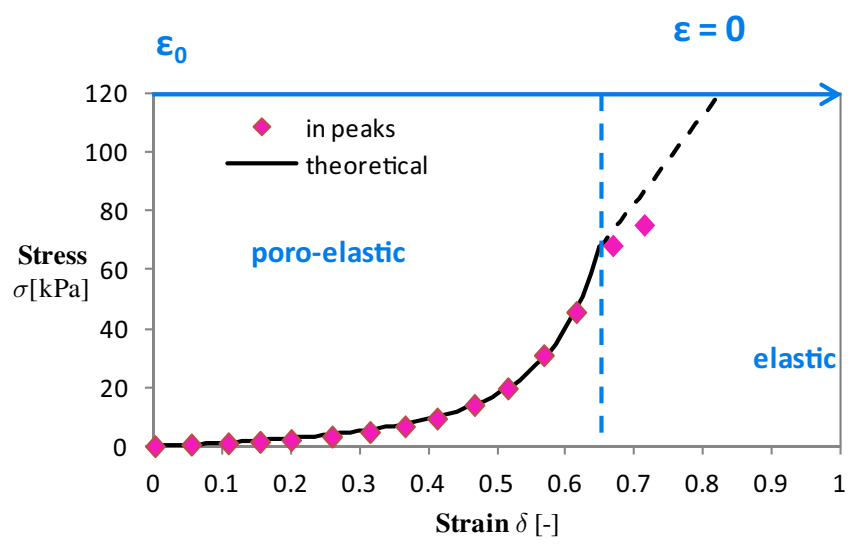

Fig. 16. Experimental curves and theoretical points of stress variation with strain for Material C.

A summary of the tested materials is presented in Table 1 .

\subsection{Tests regarding the Maxwell model for visco-elasticity}

We assume that when the material is compressed, there is an elastic effect, due to the elasticity of the material and a viscous effect due to the flow of the fluid through the pores, neglecting that the skeleton of the material can be viscoelastic.

One can build up a model of linear viscoelasticity by considering combinations of the linear elastic spring and the linear viscous dashpot. Many complex materials, like polymers, do not relax with a single relaxation time. When considered necessary to incorporate the effect of more relaxation times, the Wiechert model (also known as the generalized Maxwell) can have many spring-dashpot Maxwell elements needed to approximate the distribution satisfactorily. The absence of the isolated dash-pot would ensure an instantaneous response. Similar behaviour of stress relaxation of chicken fibrinogen with three initial strain levels were fitted with generalized Maxwell model in reference [9].

The equation used for fitting the experimental results is a Maxwell model approximated with only 3 Prony series terms:

$$
\begin{aligned}
\frac{\sigma(t)}{\sigma_{0}}= & 1-\frac{\sigma_{1}}{\sigma_{0}}\left(1-\mathrm{e}^{-\left(t-t_{0}\right) / \lambda_{1}}\right)-\frac{\sigma_{2}}{\sigma_{0}}\left(1-\mathrm{e}^{-\left(t-t_{0}\right) / \lambda_{2}}\right) \\
& -\frac{\sigma_{3}}{\sigma_{0}}\left(1-\mathrm{e}^{-\left(t-t_{0}\right) / \lambda_{3}}\right)
\end{aligned}
$$

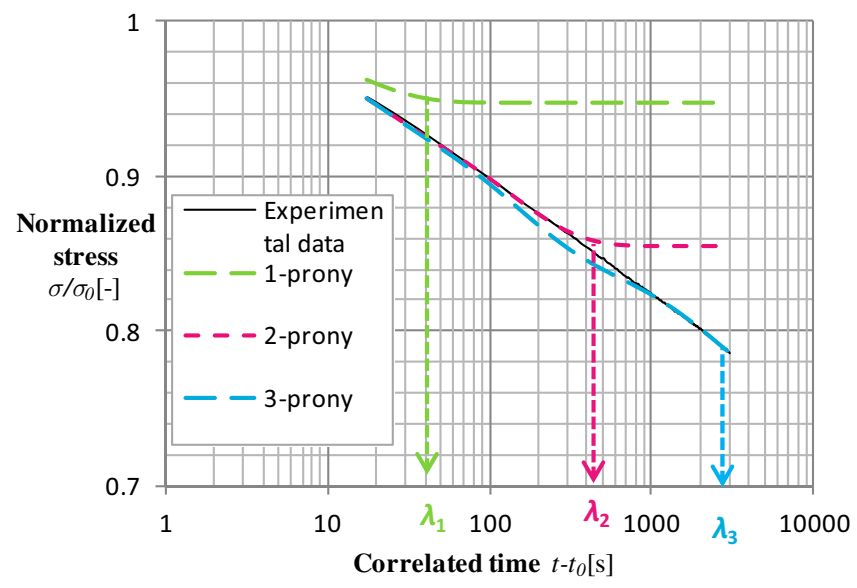

Fig. 17. Normalized stress with respect to correlated time for experiment and its approximations for Material A.

In Figure 17 a compression test of Material A up to $\delta=0.26$ strain and a long relaxation curve of $3000 \mathrm{~s}$ is presented. This curve was approximated using the Genetic algorithm with one, two or three Prony terms, named "1/2/3 - prony". The relaxation curve of max $300 \mathrm{~s}$ can be approximated well using two terms Prony series, while the approximation of a $3000 \mathrm{~s}$ curve needs the third term.

The fitted curve with three Prony terms is equivalent in terms of elastic modulus because $\frac{\sigma(t)}{\sigma_{0}}=\frac{E(t)}{E_{0}}$. Taking the elastic modulus calculated above, as $E_{0}=70 \mathrm{kPa}$, the algorithm gives: $E_{1}=3.71 \mathrm{kPa}, E_{2}=6.44 \mathrm{kPa}, E_{3}=$ $6.93 \mathrm{kPa}$ corresponding to the relaxation times of $\lambda_{1}=$ $13 \mathrm{~s}, \lambda_{2}=135 \mathrm{~s}$ and $\lambda_{3}=2680 \mathrm{~s}$.

\section{Discussions and conclusions}

The studies on porous materials imbibed with liquids developed in the paper herein regarding the viscoelastic characteristics of porous materials are trying to bring new information to the table regarding the behaviour under squeeze process at constant speed.

Viscoelasticity is confirmed by the material relaxation when kept at constant strain as long as there are open pores. The relaxation depends on the gap, due to the porosity. We can observe two types of relaxation curves of the stress: for a positive range of porosity in the interval $\varepsilon \in\left(0, \varepsilon_{0}\right)$ the stress has the tendency to decrease after a curve that seems to be going asymptotically to zero; when 
$\varepsilon=0$, the stress decreases slightly at the beginning, but then recovers close to its initial value - which is a clear proof of a purely elastic behaviour. The relaxation curves for different imbibition levels prove that the relaxation is due to the matrix or yarns relaxing in the material and not because of the water inside. The relaxation is actually faster with less water imbibed in the material, in identical compression conditions. The stress relaxes more quickly with a higher velocity, which can explain the damping capacity of these materials imbibed with water. A more intuitive result that supports this capacity is the hysteresis curve.

The stress-strain curve has to be divided into two zones, delimited by the zero-porosity limit $h_{\min }$. In the poro-elastic zone, $h \in\left(h_{\min }, h_{0}\right)$, the exponential variation of stress with respect to strain is defined as Spriggs' model - an exponential model for pores with arbitrary shape. For the elastic zone, $h \in\left(0, h_{\min }\right)$, the variation of stress is expressed as the sum of a residual stress that is calculated as the sum of the stress of the porous material at $h_{\min }(\varepsilon=0)$ and a Hookean stress of a "new" sample, elastic, of thickness $h_{\min }$.

The mathematical expressions of the stress-strain variation for the two domains (poro-elastic and elastic) are applicable to other similar materials, with different values for the elastic modulus of the fibers, $E_{0}$ and exponent, $B$.

Acknowledgements. The authors would like to thank LaMCoS (Laboratoire de Mécanique des Contacts et des Structures) of INSA Lyon (Institut National des Sciences Appliquées de Lyon) for the experimental support and guidance.

\section{References}

[1] M.D. Pascovici, Procedure and device for pumping by fluid dislocation, Romanian patent No. 109469, 1994

[2] J. Feng, S. Weinbaum, Lubrication theory in highly compressible porous media: the mechanics of skiing, from red cells to humans, J. Fluid Mech. 422 (2000) 281-317

[3] R. Crawford, R. Nathan, L. Wang, Q. Wu, Experimental study on lift generation inside a random synthetic porous layer under rapid conpaction, Exper. Therm. Fluid Sci. 36 (2012) 205-216

[4] M. Nabhani, M. El Khlifi, B. Bou-Saïd, A numerical simulation of viscous shear effects on porous squeeze film using the Darcy-Brinkman model, Mechanics \& Industry 11 (2010) 327-337

[5] M. Nabhani, M. El Khlifi, B. Bou-Saïd, A generalized model for porous flow in squeezing film situations, Lubr. Sci. 22 (2010) 37-53

[6] B. Ilie, Damping capacity of highly compressible porous layers imbibed with liquids for cylindrical configurations, Ph.D. Thesis, 2011

[7] R.M. Spriggs, Expression for Effect of Porosity on Elastic Modulus of Polycrystalline Refractory Materials, Particularly Aluminum Oxide, J. Am. Ceramic Soc. 44 (1961) 628-629

[8] E. Gregorova, W. Pabst, Effective elastic properties of alumina-zirconia composite ceramics, Ceramics Silikáty 48 (2004) 165-174

[9] J.C. Wang, Young's modulus of porous materials. Theoretical derivation of modulus-porosity correlation, J. Mater. Sci. 19 (1984) 801-808

[10] J. Hou, X. Xu, A stress relaxation model for the viscoelastic solids based on the stead-state creep equation, Mech. Time-Depend Mater 15 (2011) 29-39 